\title{
Railway Hospital Rises to the Occasion: A High Risk Moderately Severe Covid 19 Case Managed
}

\section{Govindarajugari Supritha Bai ${ }^{1 *}$ and Suresh Kishanrao ${ }^{2}$}

${ }^{1} B P T$, (MPH), Karnataka State Rural Development and Panchayat Raj University, India

${ }^{2}$ Public Health Consultant \& Visiting Professor-MPH, India

*Corresponding Author: Govindarajugari Supritha Bai, BPT, (MPH), Karnataka

State Rural Development and Panchayat Raj University, India
Received: July 26, 2021;

Published: August 23, 2021

(C) All rights are reserved by Govindarajugari

Supritha Bai and Suresh Kishanrao.

\section{Abstract}

Coronavirus disease 2019 (COVID-19) is a respiratory tract infection caused by a newly emergent coronavirus, called SARC Covid-19. The first 3 cases of COVID-19 in India were reported in the towns of Thrissur, Alappuzha, and Kasaragod, of Kerala, among Indian medical students who had returned from Wuhan. Lockdown was announced in Kerala on $23^{\text {rd }}$ March and subsequently in the rest of the country. COVID-19 had a detrimental impact on urban population initially, as the virus has caused hundreds of thousands of deaths. The second phase of COVID-19 since April 2021 has been having devasting effects both in cities and spreading in rural area. Major Indian cities like Mumbai, Delhi, Pune, and Bengaluru, faced a lot of problem as they had run around for hospital beds, drugs, oxygen etc. This was the time every health facility in the cities was roped in the management of cases. I report here a case of a 40 years, railway employee, male diabetic, and obese reporting to a Railway hospital, with the complaints of severe body pain, headache, weakness, sore throat, and breathlessness for 7 days. He tested positive for RT-PCR for corona virus disease. In the first week he had mild symptoms, but in the second week his temperature and breathlessness became severe, compelled him to seek consultation and he was admitted in hospital. Being an employee of the railways, living in the railway quarters is entitled for free treatment, therefore he sought the care there. Railway hospitals provide safe and scientific health services to maximum number of our fellow citizen. He was kept under ventilator and given oxygen, oral medications like Oseltamivir $75 \mathrm{mg}$ twice daily for two days, aziwok $500 \mathrm{mg}$ once a day for five days and HCQS $400 \mathrm{mg}$ twice daily for one day and $200 \mathrm{mg}$ twice a day for four days and for comorbidity of diabetes was given metformin $500 \mathrm{mg}$ twice a day, gluformin $1 \mathrm{mg}$ twice a day, teneligliptin $20 \mathrm{mg}$ once a day (taking in the last 6 months) and with good diet. After 5 days he was weaned off the ventilator and then shifted to general ward and after few days he was discharged and advised for follow-up. At home he followed regular medications, physical activities and followed low carb diet for diabetes and recovered from COVID-19 and is currently normally since $20^{\text {th }}$ April 2021 . He has long term post COVID symptoms like body pains and tiredness even after 6 weeks now.

Keywords: COVID-19; Diabetes; RT-PCR; Obesity; Railway Hospital

\section{Background}

Coronavirus disease (COVID-19) is an infectious disease caused by a newly discovered coronavirus, most people affected with the COVID-19 virus will experience mild to moderate respiratory ill- ness and recover without requiring special treatment. Older people, and those with underlying medical problems like cardiovascular disease, chronic respiratory disease, and cancer are more likely to develop serious illness. 
The catastrophic second wave of COVID-19 ravaging the country has left Bengaluru battered, with its active caseload hovering over 3.5 lakhs on May $9^{\text {th }} 2021$, the figure is three times that of Delhi and six times that of Mumbai. Irrefutably, the worst affected metro city in the second wave, Bengaluru has left its citizens scrambling for beds, oxygens and other medical supplies. BBMP has taken over the responsibility of allotting beds even in private hospitals. Many hospitals in Bengaluru stopped admitting COVID-19 patients directly. Shortage of anti-viral drugs, oxygen and other supportive equipment like thermometers and Pulse oximeters had become scarce commodities. Sustaining the fight against COVID-19 the Indian railways made an all-out effort to supplement the health care efforts of Government of India. Railway hospitals were converted into COVID hospitals by ensuring provision of ventilators, $\mathrm{O}_{2}$ cylinders, arrangement for disposal of biological wastes, dedicated separate entry and exit [1]. Indian railway hospitals have a very well-placed administrative hierarchy from top office to field level, with well planned, organized, motivated, coordinated and controlled health care delivery system with vast infrastructure and skilled and committed man power resources. The Indian Railways Health system set up has a total 586 health units and 125 Railway hospitals having 13963 indoor beds, 2506 doctors and 54337 health care workers. The health care services of railway hospitals are responsible for providing comprehensive health care to the serving railway employees, retired railway employees and their dependents as well as other categories of staff such as contractors, vendors and licensed porters. Indian Railways adopted multipronged strategy to fight COVID-19 by mid-May 2020. They have deployed more than 2500 doctors and 35000 paramedic staff to meet the COVID-19 challenge in a phased manner. In Railway hospitals around 5000 beds in 17 dedicated hospitals and 33 hospital blocks have been identified and dedicated for treatment of COVID-19 patients. The health care facilities of Indian railways are spread all over the country to serve railway beneficiaries. It has a three-tier system of health care facilities which includes health units meant for providing primary level health care, sub-divisional and divisional hospitals with specialty services for providing secondary health care, and then there are tertiary level health care facilities with specialty and super-specialty care. Railway board has designated some railway hospitals as Dedicated COVID-19 Hospitals or Dedicated COVID Block of railway hospitals. As per guidelines of MoH\&FW, GoI, Railway board designated certain beds in identified facilities for care of COVID patients in Railway hospitals [2].

\section{Introduction}

The novel coronavirus 2019 (COVID-19) also known as severe acute respiratory syndrome coronavirus 2 (SARS-CoV-2) is an enveloped, non-segmented positive-sense RNA virus belonging to the beta-coronaviridae family [1]. The World Health Organization declared it as a Public Health Emergency of International Concern on 30 January 2020, and later declared it as a pandemic on 11 March 2020 [2]. As of $1^{\text {st }}$ June, more than 170 million cases have been confirmed, with more than 3.55 million confirmed deaths, it is declared as one of the deadliest pandemics in history.

As per MOH and FW GOI, India from 3 January 2020 to July 7, 2021, had 30,663,665 confirmed cases of COVID-19 with 404,211 deaths, highest number of cases in a day were 4,14,188 reported on 7th may 2021 and since then there is a continuous decline in daily cases. Currently, India reports $11 \%$ of new COVID cases reported globally and 32 daily cases per million population [3]. In Karnataka confirmed cases are 28,62,338 and deaths are 35,601 and in Bangalore Urban there are 1217507 confirmed cases and 15702 deaths [4].

Symptoms of coronavirus are highly variable, ranging from none to life-threateningly severe, COVID-19 affects different people in different ways, most infected people will develop mild to moderate illness and recover without hospitalization. Most common symptoms are fever, dry cough, tiredness, and less common symptoms are aches, sore throat, diarrhea, conjunctivitis, headache, loss of taste or smell and serious symptoms includes difficulty breathing or shortness of breath, chest pain or pressure, loss of speech or movement. It is transmitted when people breathe in air contaminated by droplets and small airborne particles, person to person close contact, and touching surface or object with the virus on it. Preventing measures include social distancing, wearing face masks in public and ventilation and air filtering, hand washing, covering one's mouth when sneezing or coughing, disinfecting surfaces and monitoring and self-isolation for people exposed or symptomatic and vaccines have also been taken.

\section{Case Presentation}

A 40-year-old railway employee, from Bangalore male, with a medical history of obesity for 4 years and diabetes past 6 months reported symptoms of severe body pains, headache, weakness, sore throat, and breathlessness admitted in Railway hospital in 
Bangalore. The Railways Health system follows the MOH and FW, GOI guidelines for all national program implementations. The guidelines provided by Railway hospitals are to observe the patients' symptoms and clinical condition, monitor Temperature, pulse rate, Respiratory rate and SPO2. The primary health care providers refer the case in case of worsening of the symptoms/clinical conditions, to the designated center for further management. The Railways gave wide publicity for preventive steps like using mask, keeping safe distance, avoiding going into the crowds and frequent washing of hands with soap and water etc. or using disinfectants when that was not possible, the standard precautionary measures of COVID pandemic advocated by national authorities. One week ago the patient had developed mild symptoms like fever, tiredness and mild headache, after 3 days he developed sore throat and body aches, in home he used medications and self-healing treatment like paracetamol, Vit-C, steaming and multivitamins up to 7 days, on the $7^{\text {th }}$ day he developed symptoms like body pains, severe head ache and breathlessness, on that day he was tested RT-PCR positive for corona virus disease and next day on $8^{\text {th }}$ he got admitted in the Railway hospital by himself, as it is close by his house in railway quarters on $2 / 4 / 2021$.

\section{Physical exam}

The findings noted by doctors are: a) Temperature of $101 \mathrm{~F}, \mathrm{~b}$ ) pulse rate 98 beats per minute, c) respiratory rate of 17 beats per minute, d) $\mathrm{SPO}_{2}$ of $85 \%$, e) blood pressure $135 / 80 \mathrm{mmHg}$, f) weight $105 \mathrm{~kg}$ (BMI 37.5).

\section{Investigations}

i) $\mathrm{Hb} \%-17.4 \mathrm{gm}$, ii) CBC-5700, iii) ESR-88 mm/hr, iv) FBS-220 $\mathrm{mg} / \mathrm{dl}, \mathrm{v})$ Cholestrol-160 mg/dl, vi) D-Dimer- 211.0, vii) LDH$2085 \mathrm{IU} / \mathrm{L}$, viii) ferritin-231.2 mg/ml, ix) platelet count-1.6L, x) S.creatinine- $0.67 \mathrm{mg}$, xi) S.Bilirubin total- $0.2 \mathrm{mg}$.

\section{Treatment}

On first day of hospital 2/4/2021, doctors observed reducing of saturation levels about $84 \%$ and also increased fasting BS of about $220 \mathrm{mg} / \mathrm{dL}$ and PP BS $300 \mathrm{mg} / \mathrm{dL}$, he was shifted into COVID-19 ICU ward by providing ventilator.

- $\quad$ Oseltamivir $75 \mathrm{mg}$ given it helps to stop the spread of virus in body and it is given twice a day for two days,
- Aziwok $500 \mathrm{mg}$ (contains azithromycin) it is an antibiotic, used to treat the bacterial infections of the respiratory system, once a day it is given,

- Monocef injection another antibiotic contains ceftriaxone it is given,

- HCQS (hydroxychloroquine) $400 \mathrm{mg}$ and $200 \mathrm{mg}$ were given twice in a day,

- Multivitamin is given daily once to treat the vitamins deficiency,

- Zinc $50 \mathrm{mg}$ is given daily once for maintaining a healthy immune system.

\section{Prognosis}

First 3 to 5 days saturation levels were fluctuated between 86 to 91 and on later $6^{\text {th }}$ day, it came to normal $96 \%$ and patient shifted to general COVID-19 ward kept him observation for 4 days. On 12/4/2021 patient was discharged from hospital, and they prescribed him treatment and told the patient to take home quarantine for 14 days and to follow up for treatment.

\section{Follow up}

After 15 days follow up, patient had recovered fully and was advised to take multivitamin, zinc tablets for one month and to follow a good diet without more carbs, sugars, and fat for weight loss.

Status as on 10 July 2021

He still has post-COVID symptoms like muscular ache, mild headache. His blood sugar levels are still high at FBS-170 mg/dL and PPBS-220 mg/Dl.

\section{Discussion}

COVID-19 is a serious infection that has led to thousands of cases of severe pneumonia and ARDS. Patients with obesity and diabetes are also at risk of severe COVID-19, complications, long term symptoms, increased BP and Blood sugar level and outcomes deaths across the globe [3]. As of now there is no approved treatments for the viral pandemic. Covid 19 Pandemic has led to disruption of lot many health services in India since April 2020 [4]. Various medications have shown to be effective. Standard protocol 
given for COVID-19, for mild disease of COVID-19 symptoms with fever/symptoms of upper respiratory tract infections and $\mathrm{SPO}_{2}>$ $97 \%$ on room air, treatment of home isolation, Tab HCQS $400 \mathrm{mg}$ $\mathrm{BD}$ and $200 \mathrm{mg} \mathrm{BD}$ for four days, for moderate disease symptoms of pneumonia with $\mathrm{RR}>24 / \mathrm{min}, \mathrm{SPO}_{2}<94 \%$ on room air.

Hospitalization is advised if there are comorbidities, or respiratory distress is noticed needing oxygen therapy or ventilation as was done in the reported case. Hydroxychloroquine, a well-known anti-malarial and auto-immune medication, is relatively inexpensive and has been extensively studied in the treatment for COVID-19 [6]. The role of Tab HCQS is doubted and taken off from the Indian protocol. However, in our case it was given $400 \mathrm{mg} \mathrm{BD}$ and $200 \mathrm{mg} \mathrm{BD}$ for four days following initial guidelines. As $\mathrm{SPO}_{2}$ was $<90 \%$ on room air, treatment of hospital admission with oxygen therapy via HFNO/NIV with helmet was given and then transferred to ICU and remdesivir at $200 \mathrm{mg}$ IV loading dose on day one, 100 mg IV for next 5 - 10 days given [5]. Other complimentary treatments included vitamin $\mathrm{C}$ and zinc, as they are known to improve the human immune system and aid in shortening the duration of and improving outcomes in respiratory infections including pneumonia. Azithromycin and multivitamin and mineral supplements have obtained a large attention in the treatment of COVID-19 as was followed in the reported case too.

\section{Conclusion}

We report a case of 40-years old male, diabetic, and obese adult known as high risk by national protocols, in Railways Hospital Bengaluru. The symptoms of severe body pains, fever, breathlessness, and weakness and investigations of RTPCR tested positive and poor SPO2 levels led to hospitalization. COVID-19 in an individual with prior comorbidities like diabetes required regular monitoring of vital signs. The national norms prescribe hospitalization of such patients. Post-Covid long term symptoms may prevail for few weeks as in the case reported. Dedicated Railway COVID-19 hospitals, Railway hospitals of COVID blocks are being equipped to take good care of patients along the lines of guidelines and advisory statements of Ministry of Railways, MoHFW.

\section{Take Home Messages}

- Covid 19 infection has become quite common in Indian cities.
- $\quad$ Though majority of cases are either asymptomatic or mild, 15 - 20\% cases need hospitalizations and $5 \%$ may need oxygen ventilator support for a few days.

- $\quad$ Persons with comorbidities like diabetes, hypertension, COPD are at higher risk and need to be managed in hospitals.

- $\quad$ All varieties of health facilities in the country are doing yeomen service in managing cases.

\section{Bibliography}

1. Trivedi L. "Combating COVID-19 pandemic: The role of indian railways”. Apollo Medicine 17.3 (2020): 235-236.

2. Patel CK., et al. "Railway anaesthesiologists and Indian railway COVID-19 management system". Indian Journal of Anesthesia 64 (2020): S132.

3. Al-Sabah S., et al. "COVID -19: Impact of obesity and diabetes on disease severity". Clinical Obesity 10.6 (2020).

4. K Suresh. "Impact of COVID-19 Pandemic on Integrated Management of Diabetes in India in 2020". 3.5 (2021): 24-31.

5. Parasher A. "COVID-19: Current understanding of its Pathophysiology, Clinical presentation and Treatment". Postgraduate Medical Journal 97.1147 (2021): 312-320.

6. Douedi S and Miskoff J. "Novel coronavirus 2019 (COVID-19)”. Medicine (Baltimore) 99.19 (2020): e20207.

7. Zhao W., et al. "A systematic approach is needed to contain COVID-19 globally". Science Bulletin 65.11 (2020): 876-878.

\section{Volume 3 Issue 9 September 2021 (C) All rights are reserved by Govindarajugari Supritha Bai and Suresh Kishanrao.}

